\title{
RISK FACTORS FOR AND MORTALITY OF EXTENDED-SPECTRUM- $\beta$-LACTAMASE-PRODUCING Klebsiella pneumoniae AND Escherichia coli NOSOCOMIAL BLOODSTREAM INFECTIONS
}

Silvana Vargas SUPERTI(1), Gustavo AUGUSTI(2) \& Alexandre Prehn ZAVASCKI(3)

\begin{abstract}
SUMMARY
A case-control study, involving patients with positive blood cultures for Klebsiella pneumoniae (KP) or Escherichia coli (EC) $\mathrm{EC}$ and controls with positive blood cultures for non-ESBL-KP or EC, was performed to assess risk factors for extended-spectrum- $\beta$ lactamase (ESBL) production from nosocomial bloodstream infections (BSIs). Mortality among patients with BSIs was also assessed. The study included 145 patients (81, 59.5\% with $K$. pneumoniae and 64, 44.1\% with E. coli BSI); 51 (35.2\%) isolates were ESBL producers and 94 (64.8\%) nonproducers. Forty-five $(55.6 \%)$ K. pneumoniae isolates were ESBL producers, while only six (9.4\%) $E$. coli isolates produced the enzyme. Multivariate analysis showed that recent exposure to piperacillin-tazobactam (adjusted Odds Ratio [aOR] 6.2; 95\% CI 1.1-34.7) was a risk factor for ESBL BSI. K. pneumoniae was significantly more likely to be an ESBL-producing isolate than $E$. coli (aOR 6.7; 95\%CI 2.3-20.2). No cephalosporin class was independently associated with ESBLs BSI; however, in a secondary model considering all oxymino-cephalosporins as a single variable, a significant association was demonstrated (aOR 3.7; 95\% CI 1.3-10.8). Overall 60-day mortality was significantly higher among ESBL-producing organisms. The finding that piperacillintazobactam use is a risk factor for ESBL-production in KP or EC BSIs requires attention, since this drug can be recommended to limit the use of third-generation cephalosporins.
\end{abstract}

KEYWORDS: Escherichia coli; Klebsiella pneumoniae; Extended-spectrum-beta-lactamase; Bacteremia; Bloodstream infection; Drug resistance; Bacterial; Risk factors.

\section{INTRODUCTION}

Klebsiella pneumoniae and Escherichia coli are leading causes of nosocomial infections, notably pneumonia and urinary tract infection, and common bloodstream infections (BSIs) ${ }^{1}$. Extended-spectrum- $\beta$ lactamases (ESBLs) have emerged worldwide as a major determinant of antimicrobial penicillin, cephalosporin and aztreonam resistance among these Gram-negative bacteria ${ }^{19}$. As occurs in many Brazilian hospitals $^{7,14,16}$, ESBL-producing organisms are endemic and highly prevalent in our institution.

Some investigations have shown that ESBL production adversely affects important clinical outcomes of patients with Enterobacteriaceae BSI, including mortality ${ }^{22}$. The identification of exposures associated with infection by resistant pathogens has been attempted in order to potentially correct modifiable variables and to improve knowledge regarding both the epidemiology and pathogenesis of resistant organisms ${ }^{26}$. Additionally, the identification of risk factors may help clinicians in their choices of empirical antibiotic therapy for individual patients, potentially resulting in better outcomes for patients with such infections.

Although risk factors for ESBL have been frequently explored in the literature, fewer studies have focused on the identification of risk factors for ESBL in patients with BSIs ${ }^{4,6,10,11,13,15,17,20-24}$. However, these studies usually consider antibiotic exposure as a single variable and/or have not performed multivariate analysis. Thus, very few studies have appropriately investigated the association of each antibiotic class or specific antibiotic with ESBL production by bloodstream $K$. pneumoniae and $E$. coli isolates ${ }^{6,15,21,24}$. The aim of this study was to assess risk factors for ESBL producing K. pneumoniae (ESBL-KP) and E. coli (ESBL-EC) nosocomial BSI, with particular focus on the role of distinct antibiotic classes. A secondary goal was to compare the mortality of patients with ESBL producing organisms BSI with those with non-ESLB pathogens.

\section{MATERIAL AND METHODS}

A case-control study was performed in a 600-bed tertiary-care teaching hospital in Porto Alegre, Southern Brazil. The Laboratory of Microbiology database was used to search for cases. Those involving positive blood cultures for K. pneumoniae and E. coli, from June 2004 to March 2006, were eligible for the study. Patients were excluded when they were less than or equal to 18 years-old and when blood culture was collected less than 48 hours after hospital admission, unless the patient had been hospitalized within the previous 60 days. Data were collected

(1) Laboratory of Microbiology, Hospital São Lucas da Pontifícia Universidade Católica do Rio Grande do Sul, Porto Alegre, RS, Brazil.

(2) Dept. Internal Medicine, Hospital São Lucas da Pontifícia Universidade Católica do Rio Grande do Sul, Porto Alegre, RS, Brazil.

(3) Infectious Diseases Service, Hospital São Lucas da Pontifícia Universidade Católica do Rio Grande do Sul, Porto Alegre, RS, Brazil.

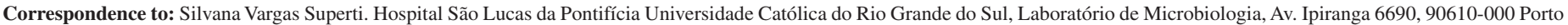
Alegre, RS, Brasil. Tel./Fax: +55.51.3320-3145. E-mail: silvanas@ufcspa.edu.br 


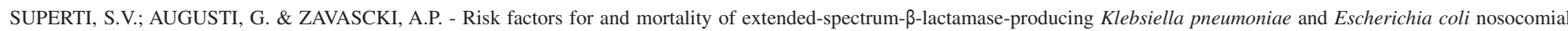
bloodstream infections. Rev. Inst. Med. trop. S. Paulo, 51(4): 211-216, 2009.

from medical records and/or hospital computer system databases. The primary site of infection was defined according to CDC criteria ${ }^{8}$. The hospital's Ethics Review Board approved this study. Written informed consent was not required because it was a retrospective study.

The isolates were identified by conventional microbiological methods and commercial systems, including API20E ${ }^{\circledR}$ (bioMérieux) and AutoScan $-4^{\circledR}$ (Dade Behring). Antimicrobial susceptibilities were determined by disk diffusion, according to Clinical Laboratory Standards Institute (CLSI) recommendations ${ }^{3}$. All K. pneumoniae and E. coli isolates were screened for potential ESBL production using screening breakpoints proposed by the CLSI, with ceftazidime, cefotaxime, ceftriaxone and aztreonam disks. For phenotypic confirmatory test, Mueller-Hinton agar plates (bioMérieux ${ }^{\circledR}$ ) with disks containing $30 \mu \mathrm{g}$ of ceftazidime and disks containing $30 \mu \mathrm{g}$ of cefotaxime (OXOID®), with and without $10 \mu \mathrm{g}$ of clavulanic acid were used and interpreted, as recommended by the CLSI ${ }^{3}$.

The cases were patients with nosocomial BSI by ESBL-KP or EC and controls were patients with BSI by non-ESBL-KP or EC. The variables potentially associated with the ESBL infections included: age; sex; Charlson comorbidity index score ${ }^{2}$; Pitt bacteremia score ${ }^{18}$; iatrogenic immunosuppression, such as chemotherapy-induced neutropenia (neutrophil count $<1,000$ cells $/ \mathrm{mm}^{3}$ ), and/or receipt of corticoid drugs or other immunosuppressive agents for less than 14 days; time at risk (length of hospital stay before K. pneumoniae or E. coli recovery); previous surgical procedure during hospital stay; intensive care unit (ICU) admission; mechanical ventilation; primary site of infection; presence of other infections; polymicrobial bacteremia; antimicrobial exposure (only those used for at least 48 hours during the previous 14 days were analyzed): $\beta$-lactams, including monobactams (aztreonam), first-generation cephalosporins (cefadroxil, cephalexin, cefazolin and cephalothin), second-generation cephalosporins (cefuroxime and cefuroxime axetil), cephamycins (cefoxitin), third-generation cephalosporins (ceftriaxone and ceftazidime), fourth-generation cephalosporins (cefepime), ureidopenicillins and $\beta$-lactamase inhibitor (piperacillin-tazobactam), carbapenems (ertapenem, imipenem and meropenem), fluoroquinolones (ciprofloxacin and levofloxacin), aminoglycosides (gentamicin and amikacin), anaerobicidal agents (clindamycin and metronidazole) and glycopeptides (vancomycin); and exposure to more than one of the antibiotics studied.

All statistical analyses were performed using SPSS for Windows, Version 13.0. Bivariate analysis was performed separately for each of the variables. Odds ratios (OR) and 95\% confidence intervals (95\% CI) were calculated for binomial variables. $P$ values were calculated using the $\mathrm{Chi}$ square or Fisher exact tests for categorical variables and the Student t or Wilcoxon rank-sum tests for continuous variables. Variables for which the $p$ value was $\leq 0.10$ in bivariate analysis were included one by one in a logistic regression model according their $p$ value and the magnitude of their effect. A $p$ of 0.10 was set as the limit for acceptance or removal of the new terms in the model.

Overall 60-day mortality was assessed and the relative risk (RR) was calculated. Survival curves were prepared using Kaplan-Meier estimation and compared using the log-rank test. All tests were two-tailed and $p \leq$ 0.05 was considered significant.

\section{RESULTS}

A total of 145 patients were included in the study. Eighty-one (59.5\%) isolates were K. pneumoniae and 64 (44.1\%) were E. coli. Fifty-one $(35.2 \%)$ isolates were ESBL producers and 94 (64.8\%) were nonproducers. The incidence of BSIs due to ESBL-KP or EC and nonESBL-KP or EC isolates was not significantly different for any month for either hospital throughout the study $(p=0.24)$. ESBL production was significantly more frequent among $K$. pneumoniae $(45,55.6 \%$ of the isolates) than E. coli (6, $9.4 \%$ of the isolates), $p \leq 0.001$. No isolate presented resistance to carbapenems. Resistance to any of the drugs tested was significant $(p<0.05)$ and more frequently observed in ESBL producer organisms than non-ESBL (data not shown), including second, third and fourth-generation cephalosporins, cefoxitin, piperacillintazobactam, aztreonam, ciprofloxacin and amikacin.

The results of bivariate analysis of risk factors for ESBL-KP or EC BSI are presented in Table 1. None of the patients were exposed to aztreonam or cefoxitin, so these agents are not presented in the analysis.

The results of the multivariate analysis showed that recent use of the combination antibiotic piperacillin-tazobactam (adjusted [a] OR 6.2 ; 95\% CI 1.1-34.7; $p=0.04$ ) was the most important risk factor for ESBL BSI. BSI due to $K$. pneumoniae, rather than $E$. coli, was also independently associated with ESBL (aOR 6.7; 95\%CI 2.3-20.2; $p<$ $0.001)$. Pitt bacteremia score remained in the final model, but showed no statistical significance (aOR 1.1; 95\% CI 1.0-1.3; $p=0.16$ ). Second, third or fourth-generation cephalosporins did not present statistical significance in the multivariate analysis, so none of these drugs remained in the final model. No other variable remained in the final model.

Considering a potential $\beta$ error due to low exposure to oxyminocephalosporins (i.e. second-, third- and fourth-generation cephalosporins) in both cases and controls, a secondary model was performed including all these drugs as a single variable, which were included individually in the first model. In bivariate analyses, oxymino-cephalosporins were significantly associated with ESBL BSI (OR 3.5; 95\%CI 1.5$8.2 ; p=0.007)$. The results of this second multivariate model showed that piperacillin-tazobactam (OR 6.7; 95\%CI 1.3-35.1; $p=0.03$ ) and $K$. pneumoniae (OR 8.3; 95\%CI 2.5-27.5; $p<0.001$ ) remained independently associated with ESBL BSI, as determined in the main model and that oxymino-cephalosporins (OR 3.7; 95\%CI 1.3-10.8; $p=$ 0.02) were also associated with ESBL BSI. Pitt bacteremia score was also maintained in the final model, but with no statistical significance $(p=0.12)$.

Overall 60-day mortality of patients with KP or EC BSI was $37.2 \%$ (54 of 145 patients) and was significantly higher among patients with BSI by ESBL than non-ESBL producers (26 out of 51 [51.0\%] versus 28 out of 94 patients [29.8\%], respectively, RR $1.71,95 \%$ CI $1.14-2.58$, $p=0.019$ ). The mortality rate was 26.0 per 1000 patient-days among patients with ESBL BSIs and 16.4 per 1000 patient-days among those with non-ESBL BSIs $(p=0.08)$. However, according to survival curves, mortality rates were higher, though not statistically significant ( $p=$ 0.99), among patients with non-ESBL BSIs during the first 14 days (41.6 per 1000 patient-days) than among those with ESBL BSIs (22.1 per 1000 patient-days). These rates were significantly higher among ESBL-producing organisms after this period: 22.2 per 1000 patient-days 


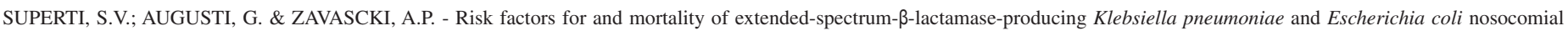
bloodstream infections. Rev. Inst. Med. trop. S. Paulo, 51(4): 211-216, 2009.

Table 1

Bivariate analysis of the risk factors for extended-spectrum- $\beta$-lactamase (ESBL)-producing Klebsiella pneumoniae and Escherichia coli nosocomial bloodstream infection

\begin{tabular}{|c|c|c|c|}
\hline Variables a & $\begin{array}{l}\text { ESBL + } \\
(n=51)\end{array}$ & $\begin{array}{l}\text { ESBL - } \\
(n=94)\end{array}$ & $p$ \\
\hline Klebsiella pneumoniae infection & $45(73.8)$ & $36(38.3)$ & $<0.001$ \\
\hline Age, years & $61.7 \pm 18.1$ & $58.7 \pm 17.4$ & 0.34 \\
\hline Sex, male & $32(62.7)$ & $49(52.1)$ & 0.29 \\
\hline Charlson score, median ( $25-75 \%$ percentile) & $3(2-6)$ & $3(2-5)$ & 0.72 \\
\hline Pitt bacteremia score, median ( $25-75 \%$ percentile $)$ & $2(0-4)$ & $0(0-2)$ & 0.04 \\
\hline Iatrogenic immunosuppression & $9(17.6)$ & $24(25.5)$ & 0.38 \\
\hline Length of hospital stay, days & $26 \pm 20$ & $16 \pm 16$ & 0.002 \\
\hline Intensive Care Unit admission & $26(51.0)$ & $27(28.7)$ & 0.01 \\
\hline Mechanical ventilation & $17(33.3)$ & $12(12.8)$ & 0.006 \\
\hline Previous surgery & $35(68.6)$ & $43(45.8)$ & 0.04 \\
\hline \multicolumn{4}{|l|}{ Site of infection } \\
\hline Lower respiratory tract & $14(27.5)$ & $11(11.7)$ & 0.03 \\
\hline Urinary tract & $7(13.7)$ & $26(27.7)$ & 0.09 \\
\hline Skin and soft tissue & $0(0)$ & $1(1.1)$ & 0.65 \\
\hline Central venous catheter & $11(21.6)$ & $14(14.9)$ & 0.43 \\
\hline Intraabdominal & $2(4.0)$ & $8(8.5)$ & 0.25 \\
\hline Primary bloodstream & $16(31.4)$ & $32(34.0)$ & 0.89 \\
\hline Central Nervous System & $0(0)$ & $2(2.1)$ & 0.42 \\
\hline Bone & $1(2.0)$ & $0(0)$ & 0.35 \\
\hline Other infections & $14(27.5)$ & $14(14.9)$ & 0.11 \\
\hline Polymicrobial infection & $27(52.9)$ & $90(95.8)$ & 0.10 \\
\hline Presence of urinary catheter & $32(62.7)$ & $38(40.4)$ & 0.02 \\
\hline Presence of central venous catheter & $32(62.7)$ & $40(42.6)$ & 0.03 \\
\hline \multicolumn{4}{|l|}{ Antibiotic exposure } \\
\hline \multicolumn{4}{|l|}{$\beta$-lactams ${ }^{\mathrm{b}}$} \\
\hline First-generation cephalosporin & $1(2.0)$ & $3(3.2)$ & 0.41 \\
\hline Second-generation cephalosporin & $3(5.9)$ & $0(0)$ & 0.08 \\
\hline Third-generation cephalosporins & $4(7.8)$ & $2(2.1)$ & 0.11 \\
\hline Cefepime & $12(23.5)$ & $9(9.6)$ & 0.12 \\
\hline Piperacillin-tazobactam & $8(15.7)$ & $1(1.1)$ & 0.01 \\
\hline Carbapenems & $4(7.8)$ & $7(7.5)$ & 0.58 \\
\hline Fluoroquinolones ${ }^{\mathrm{c}}$ & $13(25.5)$ & $20(21.3)$ & 0.71 \\
\hline Aminoglycosides ${ }^{\mathrm{d}}$ & $1(2.0)$ & $2(2.3)$ & 0.72 \\
\hline Vancomycin & $9(17.6)$ & $8(8.5)$ & 0.55 \\
\hline Anaerobicidal ${ }^{\mathrm{e}}$ & $17(33.3)$ & $12(12.8)$ & 0.09 \\
\hline Use of more than one of the antibiotics studied ${ }^{f}$ & $17(33.3)$ & $11(11.7)$ & 0.003 \\
\hline
\end{tabular}

+, producer; -, nonproducer; ${ }^{\text {a }}$ All variables are expressed as the mean \pm SD or number (\%), unless otherwise indicated; ${ }^{b}$ First-generation cephalosporin: all 3 were cephalothin; Second-generation cephalosporin: all were 3 cefuroxime; Third-generation cephalosporins: all 6 were ceftriaxone; Carbapenems: 6 were meropenem and 5 were imipenem; ${ }^{\mathrm{c}}$ Nineteen ciprofloxacin and 14 levofloxacin; ${ }^{\mathrm{d}}$ Two amikacin and 1 gentamicin; ${ }^{\mathrm{e}}$ Twenty clindamycin and 9 metronidazole; ${ }^{\mathrm{f}}$ Fifteen $(51.8 \%)$ double exposures were to a second-, third- or cefepime and a anaerobicidal agent; $6(20.7 \%)$ were to ciprofloxacin and anaerobicidal; $3(10.3 \%)$ were to cefepime and vancomycin; 2 (6.9\%) were to a carbapenem and vancomycin; 1 (3.5\%) was to piperacillin-tazobactam and ciprofloxacin; 1 (3.5\%) was to cephalothin and ciprofloxacin; and $1(3.5 \%)$ was to ceftriaxone and ciprofloxacin. 


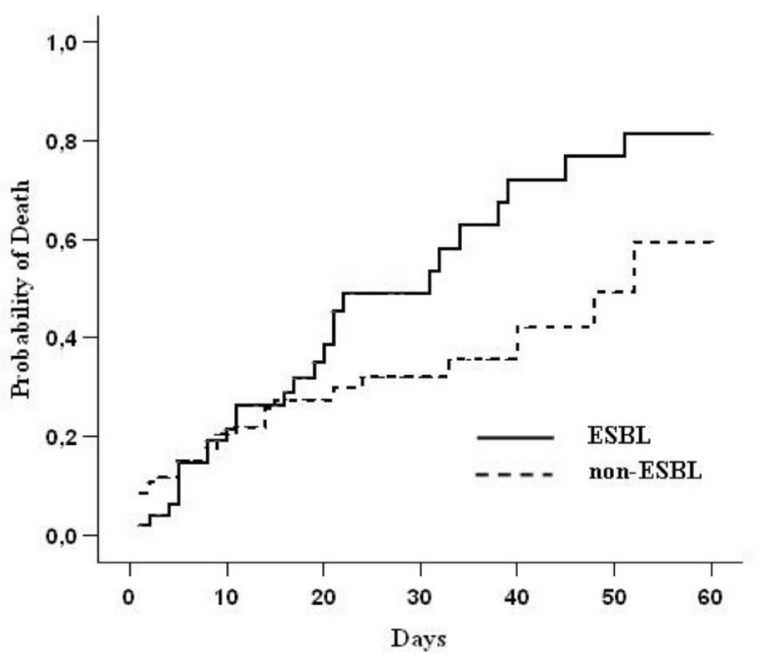

Fig. 1 - Mortality curves of patients with bloodstream infections due to extended-spectrum ß-lactamases (ESBLs) and non-ESBL-producing Klebsiella pneumoniae (KP) or Escherichia coli (EC). Curves were prepared using Kaplan-Meier estimation and compared using the log-rank test $(p=0.08)$.

among patients with ESBL BSIs and 7.1 per 1000 patient-days among those with non-ESBL BSIs $(p=0.03)$.

\section{DISCUSSION}

ESBL-producing organisms are a clinical problem worldwide, particularly in Latin American countries and especially in Brazil, where high endemic rates of ESBL-producing Enterobacteriaceae have been determined $^{7,14,16}$. In our institution, high endemic rates have also been observed over the last 10 years. The present study showed that more than half $(55.6 \%)$ of $K$. pneumoniae BSIs are caused by ESBL producers, a fact of grave concern.

This study showed that use of the combination antibiotic piperacillintazobactam within the previous 14 days was strongly associated with ESBL production in BSIs due to KP or EC. Additionally, K. pneumoniae BSIs occurred more frequently due to an ESBL-producing organism than E. coli BSIs.

Only one prior study has reported that piperacillin-tazobactam may be a risk factor for ESBL-KP or EC colonization'. The present work demonstrated a strong independent association of this combination of drugs with ESBL-KP or EC BSI; however, it should be noted that ESBL isolates presented significantly higher rates of piperacillin-tazobactam resistance than non-ESBL ones. This indicates that another resistance mechanism was probably present among ESBL producers, since ESBL is inhibited in vitro by tazobactam ${ }^{19}$. Thus, piperacillin-tazobactam exposure could actually be associated with the selection of isolates producing this other resistance mechanism, which was more frequently observed in ESBL isolates in the present study. Regardless of the cause of this association, this finding has important implications, since some antimicrobial drug stewardship policies propose an increase in the use of certain antimicrobial drugs, including piperacillin-tazobactam, and a reduction in the prescription of third-generation cephalosporins, in an effort to control ESBL-producing bacteria ${ }^{9}$.
It was not possible to demonstrate an independent association of any specific class of cephalosporin with ESBL. However, this may be due to a reduced statistical power due to the low exposure rates of each class than to the lack of a real association of the variables with the outcome, since a significant association was demonstrated when reducing $\beta$-error by analyzing all oxyimino-cephalosporins together as a single variable. Furthermore, cephalosporins, particularly oxyimino-cephalosporins, have been shown to be risk factor for ESBL in other studies ${ }^{15,19,21}$.

No association between fluoroquinolone or aminoglycoside exposure and ESBL-KP or EC BSIs was observed, as previously determined ${ }^{19}$, despite the fact that ESBL-producing organisms presented significantly more resistance to both classes of drugs than nonproducing isolates. Although the low exposure rates might explain the lack of association with aminoglycosides, this was not the case of fluoroquinolones. The use of more than one antibiotic by the same patient was statistically significant in bivariate analysis, but this was not the case in any of the regression models. We believed that this occurred because most of the double exposures $(51.8 \%)$ involved an oxyimino-cephalosporin and an anaerobicidal agent and another five (17.2\%) involved an oxyiminocephalosporin or piperacillin-tazobactam and another non-significantly associated drug (vancomycin or ciprofloxacin).

In the present study, inclusion of the causative agent of BSI, $K$. pneumoniae or E. coli, in the multivariate model was proposed in order to control potential risk factors associated with the bacteria rather than with ESBL production. The finding that $K$. pneumoniae was significantly associated with ESBL production merely reflects local and worldwide epidemiology, which clearly show that ESBL production has been more frequently observed in this bacteria than in E. coli ${ }^{19}$.

Overall 60-day mortality was significantly higher in ESBL patients than non-ESBL patients (51.0\% versus $29.8 \%$, respectively). Although not statistically significant, an important trend toward higher mortality rates among former patients was demonstrated in this study. Of note, mortality rates of patients with both ESLB and non-ESBL organisms were significantly higher after the end of the second week of the onset of BSI. The cause of this interesting finding was not assessed in this work and should be further explored. It should be noted that only crude mortality rates are presented, because this was not the main objective of the study. No adjustment for any potential confounding variables was performed and this should be considered while interpreting the results.

The study has some limitations that should be acknowledged, besides those common to all studies with a retrospective design. First, we were unable to assess the dose and duration of antimicrobial therapy, thus no potential dose-response effect could be determined. Additionally, data regarding the molecular epidemiology of ESBL$\mathrm{KP}$ or EC bloodstream isolates was not presented, neither for the characterization of the ESBL type nor for isolate typing. However, we believe that this has little or no impact on our main findings, with the exception that the role of potential horizontal-transmission could not be determined. Finally, although use of the combination antibiotic piperacillin-tazobactam was shown to be independently associated with ESBL, this finding was based on a relatively small number of observations (eight cases and one control); thus caution is required when generalizing the results, as well as additional confirmation in future studies. 
SUPERTI, S.V.; AUGUSTI, G. \& ZAVASCKI, A.P. - Risk factors for and mortality of extended-spectrum-ß-lactamase-producing Klebsiella pneumoniae and Escherichia coli nosocomial bloodstream infections. Rev. Inst. Med. trop. S. Paulo, 51(4): 211-216, 2009.

The issue of control selection in case-control studies that assess risk factors for antibiotic-resistant organisms has been matter of debate $\mathrm{f}^{5,12,25}$. Patients with non-ESBL-KP or EC BSI were chosen, because this design is well suited to assess risk factors when trying to determine which variables are associated with antibiotic resistance among patients with infections by specific organisms $\mathrm{s}^{5,12}$.

In conclusion, analysis of the results showed that exposure to piperacillin-tazobactam was a risk factor for ESBL production among $\mathrm{KP}$ or EC isolates causing BSIs. Although ESBL BSI was not associated with any specific class of cephalosporin, when considering all oxyiminocephalosporins together, an independent association was observed. Overall 60-day mortality was significantly higher among ESBLproducing bacteria, but the specific mediators of this effect and potential confounding factors were not investigated. Particular attention must be given to the potential risks of exposure to piperacillin-tazobactam, since the prescription of this drug has been recommended by some antibiotic stewardship programs in order to limit third-generation cephalosporin use. Further studies assessing the association of this specific drug with ESBL are required.

\section{RESUMO}

\section{Fatores de risco e mortalidade de infecções da corrente sanguínea por Klebsiella pneumoniae and Escherichia coli produtores de beta-lactamase de espectro estendido}

Estudo de caso-controle, onde os casos foram pacientes com hemocultura positiva para Klebsiella pneumoniae (KP) ou Escherichia coli (EC) produtores de beta lactamase de espectro estendido (ESBL) e os controles foram pacientes com hemoculturas positivas para EC ou KP não produtores de ESBL foi realizado para avaliar os fatores de risco para produção destas enzimas em infecções da corrente sanguínea (ICS). Mortalidade dos pacientes com ICS também foi avaliada. Foram incluídos 145 pacientes (81, 59,5\% tinham Klebsiella pneumoniae e 64, 44,1\% tinham Escherichia coli); 51 (35,2\%) isolados eram produtores de ESBL e $94(64,8 \%)$ eram não produtores. Quarenta e cinco $(55,6 \%)$ isolados de $K$. pneumoniae e somente seis $(9,4 \%)$ isolados de E. coli eram produtores de ESBL. Análise multivariada mostrou que exposição recente a piperacilina-tazobactam (OR ajustado [aOddsRatio] 6,2; 95\% Intervalo de Confiança [IC] 1,1-34,7) foi fator de risco para infecção da corrente sanguínea por ESBL. Foi significativamente maior a chance de $K$. pneumoniae ser um isolado produtor de ESBL do que $E$. coli o ser (aOR 6,7; 95\% CI 2,3-20,2). Nenhuma classe de cefalosporina foi independentemente associada com ESBL-ICS. No entanto, em um modelo secundário considerando todas as oximino-cefalosporinas como variável única, foi demonstrada associação significativa (aOR 3,7; 95\% IC 1,3-10,8). Mortalidade total em 60 dias foi significativamente maior entre isolados produtores de ESBL. O achado de piperacilina-tazobactam como fator de risco para produção de ESBL em ICS por KP ou EC requer atenção, uma vez que esta droga tem sido eventualmente recomendada para poupar o uso de cefalosporinas de terceira geração.

\section{REFERENCES}

1. BIEDENBACH, D.J.; MOET, G.J. \& JONES, R.N. - Occurrence and antimicrobial resistance pattern comparisons among bloodstream infection isolates from the SENTRY Antimicrobial Surveillance Program (1997-2002). Diagn. Microbiol. infect. Dis., 50: 59-69, 2004.
2. CHARLSON, M.E.; POMPEI, P.; ALES, K.L. \& MACKENZIE, C.R. - A new method of classifying prognostic comorbidity in longitudinal studies: development and validation. J. Chronic Dis., 40: 373-383, 1987.

3. CLINICAL AND LABORATORY STANDARDS INSTITUTE - Performance standards for antimicrobial susceptibility testing. Wayne, 2007. (17 ${ }^{\text {th }}$ Informational Supplement. CLSI Document M-100 S17).

4. CORDERY, R.J.; ROBERTS, C.H.; COOPER, S.J.; BELLINGHAN, G. \& SHETTY N. - Evaluation of risk factors for the acquisition of bloodstream infections with extended-spectrum beta-lactamase-producing Escherichia coli and Klebsiella species in the intensive care unit; antibiotic management and clinical outcome. J. Hosp. Infect., 68: 108-115, 2007.

5. D'AGATA, E.M. - Methodologic issues of case-control studies: a review of established and newly recognized limitations. Infect. Control Hosp. Epidem., 26: 338-341, 2005

6. DU, B.; LONG, Y.; LIU, H. et al. - Extended-spectrum beta-lactamase-producing Escherichia coli and Klebsiella pneumoniae bloodstream infection: risk factors and clinical outcome. Intensive Care Med., 28: 1718-1723, 2002.

7. GALES, A.C.; BOLMSTRÖM, A.; SAMPAIO, J.; JONES, R.N. \& SADER, H.S.Antimicrobial susceptibility of Klebsiella pneumoniae producing extended-spectrum beta-lactamase (ESBL) isolated in hospitals in Brazil. Braz. J. infect. Dis., 1: 196-203, 1997.

8. GARNER, J.S.; JARVIS, W.R.; EMORI, T.G.; HORAN, T.C. \& HUGHES, J.M. - CDC definitions for nosocomial infections, 1988. Amer. J. Infect. Control, 16: 128-140, 1988.

9. HARRIS, A.D.; McGREGOR, J.C.; JOHNSON, J.A. et al.- Risk factors for colonization with extended-spectrum beta-lactamase-producing bacteria and intensive care unit admission. Emerg. infect. Dis., 13: 1144-1149, 2007.

10. HO, P.L.; CHAN, W.M.; TSANG, K.W.; WONG, S.S. \& YOUNG, K. - Bacteremia caused by Escherichia coli producing extended-spectrum beta-lactamase: a case-control study of risk factors and outcomes. Scand. J. infect. Dis., 34: 567-573, 2002

11. KANG, C.I.; KIM, S.H.; KIM, D.M. et al.- Risk factors for and clinical outcomes of bloodstream infections caused by extended-spectrum beta-lactamase-producing Klebsiella pneumoniae. Infect. Control Hosp. Epidem., 25: 860-867, 2004.

12. KAYE, K.S.; HARRIS, A.D.; SAMORE, M. \& CARMELI, Y. - The case-case-control study design: addressing the limitations of risk factor studies for antimicrobial resistance. Infect. Control Hosp. Epidem., 26: 346-351, 2005.

13. KIM, B.N.; WOO, J.H.; KIM, M.N.; RYU, J. \& KIM, Y.S. - Clinical implications of extended-spectrum beta-lactamase-producing Klebsiella pneumoniae bacteraemia J. Hosp. Infect., 52: 99-106, 2002.

14. MARRA, A.R.; WEY, S.B.; CASTELO, A. et al. - Nosocomial bloodstream infection caused by Klebsiella pneumoniae: impact of extended-spectrum beta-lactamase (ESBL) production on clinical outcome in a hospital with high ESBL prevalence. BMC Infect. Dis., 6: 24, 2006.

15. MARTÍNEZ, J.A.; AGUILAR, J.; ALMELA, M. et al. - Prior use of carbapenems may be a significant risk factor for extended-spectrum beta-lactamase-producing Escherichia coli or Klebsiella spp. in patients with bacteraemia. J. Antimicrob. Chemother., 58: 1082-1085, 2006

16. MARTINS, I.S.; PESSOA-SILVA, C.L.; NOUER, S.A. et al. - Endemic extendedspectrum beta-lactamase-producing Klebsiella pneumoniae at an intensive care unit: risk factors for colonization and infection. Microb. Drug Resist., 12: 50-58, 2006.

17. PANHOTRA, B.R.; SAXENA, A.K. \& AL-GHAMDI, A.M. - Extended-spectrum betalactamase-producing Klebsiella pneumoniae hospital acquired bacteremia. Risk factors and clinical outcome. Saudi med. J., 25: 1871-1876, 2004. 
SUPERTI, S.V.; AUGUSTI, G. \& ZAVASCKI, A.P. - Risk factors for and mortality of extended-spectrum- $\beta$-lactamase-producing Klebsiella pneumoniae and Escherichia coli nosocomial bloodstream infections. Rev. Inst. Med. trop. S. Paulo, 51(4): 211-216, 2009.

18. PATERSON, D.L.; KO, W.C.; VON GOTTBERG, A. et al. - Antibiotic therapy for Klebsiella pneumoniae bacteremia: implications of production of extended-spectrum beta-lactamases. Clin. infect. Dis., 39: 31-37, 2004.

19. PATERSON, D.L. \& BONOMO, R.A. - Extended-spectrum beta-lactamases: a clinical update. Clin. Microbiol. Rev., 18: 657-686, 2005.

20. PEÑA, C.; PUJOL, M.; ARDANUY, C. et al. - An outbreak of hospital-acquired Klebsiella pneumoniae bacteraemia, including strains producing extended-spectrum beta-lactamase. J. Hosp. Infect., 47: 53-59, 2001.

21. RODRÍGUEZ-BAÑO, J.; NAVARRO, M.D.; ROMERO, L. et al. - Risk-factors for emerging bloodstream infections caused by extended-spectrum beta-lactamaseproducing Escherichia coli. Clin. Microbiol. Infect., 14: 180-183, 2008.

22. SCHWABER, M.J. \& CARMELI, Y. - Mortality and delay in effective therapy associated with extended-spectrum beta-lactamase production in Enterobacteriaceae bacteraemia: a systematic review and meta-analysis. J. Antimicrob. Chemother, 60: 913-920, 2007.
23. TUMBARELLO, M.; SPANU, T.; SANGUINETTI, M. et al. - Bloodstream infections caused by extended-spectrum-beta-lactamase-producing Klebsiella pneumoniae: risk factors, molecular epidemiology, and clinical outcome. Antimicrob. Agents. Chemother., 50: 498-504, 2006.

24. ZAOUTIS, T.E.; GOYAL, M.; CHU, J.H. et al.- Risk factors for and outcomes of bloodstream infection caused by extended-spectrum beta-lactamase-producing Escherichia coli and Klebsiella species in children. Pediatrics, 115: 942-949, 2005.

25. ZAVASCKI, A.P. - Assessing risk factors for acquiring antimicrobial-resistant pathogens: a time for a comparative approach. Clin. infect. Dis., 39: 871-872, 2004.

26. ZAVASCKI, A.P.; BARTH, A.L.; GASPARETO, P.B. et al. - Risk factors for nosocomial infections due to Pseudomonas aeruginosa producing metallo-beta-lactamase in two tertiary-care teaching hospitals. J. Antimicrob. Chemother., 58: 882-885, 2006.

Received: 12 January 2009

Accepted: 5 May 2009 